ansinting to correct the mal-position, by wouring-to borrow a nantical phrase- the head down to and into the pelvic cavity, cimultaneously with the breech being urged upwards to the fundus? In answer to both questions, with extreme deference, I think not; and should another arm-presentation case ever come in my way, under suitable circumstances, I will desire to put in practice the very same course, modified only according to variety in position of the child, to be presently noticed, which I unqualifiedly believe has been successful in this occasion.

Two inquiries suggest themselves. 1. Would the same manipulations produce a like result, if the child's right arm presented, its face and abdomen corresponding with those of the mother, the head still resting upon, or above her right ilium? In that case, I should proceed in precisely the manner described, and hope for a similar termination. 2. Would the same management be applicable, if the longitudinal position of the child in utero were reversed from that in the instance related, the presentation being, then, the right or left arm, but with the head, whether occiput or sinciput, situated on, or above, the mother's left ilium, and the breech in the direction of her right ilium ? Yes : but it would be necessary that the woman should lie upon her right side, and that the repressing force be employed by the right hand of the accoucheur.

Leeds, Sept. 10, 1852.

\title{
ON THE PATHOLOGY AND TREATMENT OF SOME OBSCURE CASES OF LONG-CONTINUED PAIN IN BONE.
}

By HENRY LEE, Esq., F.R.C.S., Surgeon to the Iock Hospital, eto.

The pain which attends suppuration in the interior of a bone, is not always in proportion to the pressure exercised by the confined fluid. It arises, apparently, in some cases from the tension produced in the condensed bone by the interstitial deposit of fresh bony matter. In other instances, a very small quantity of matter contained in soft spongy bone becomes a source of irritation, and appears to determine to, and fix in, the part some pain depending upon constitutional causes, and which, were it not for the disease in the bone, might have fallen upon some other region. The morbid sensations once established will continue, although not originally produced by the local disease, until that is removed; and even after it has been removed they will, as if from confirmed habit, shew a tendency to return to their accustomed place. After a time, however, if the cause which has determined the pain to a particular spot be removed, and no fresh source of irritation be present, the symptoms will cease. The piece of bone from which the accompanying.wood-cut is taken, was remored from the tibia of a person of highly nervous temperament, who had for years suffered most severe local pain, and been subject to a great variety of treatment under different medical men. At length the operation of tre- 
phining the tibia was performed by Sir B. Brodie. The pain did not immediately cease, but after an interval the patient permanently re-

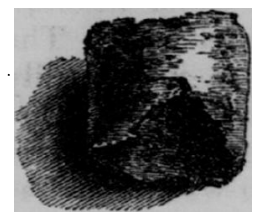

Fig. 5. Portion of thickened but soft bone, removed from a lower extremity of tibia, shewing the cavity of a small abscess.

covered. The drawing (for which I am indebted to the kindness of Mr. Charles Hawkins) shows how small the cavity was in which the fluid was contained, and the soft and spongy nature of the bone in its immediate neighbourhood.

The following case appears to show, that a constitutional affection produced by syphilis or mercury, may in like manner be determined to, and localised in, a particular bone; and that the symptoms may there be kept up for almost an unlimited time by a very small quantity of matter in its interior.

CASE III. ${ }^{2}$ A married woman, of a light complexion, and twenty-four years of age, was admitted an out-patient at King's College Hospital in the year 1849. The left knee-joint was at that time enlarged and painful, a puffy elastic swelling presented itself on the outer side of the joint, affording somewhat the appearance of a dislocation of the patella : very little fluctuation could be detected within the synovial membrane. The whole of the surrounding parts were painful, but the pain appeared to be of a very different character, and much more severe when the weight of the body rested upon the affected limb, or when the knee-joint was bent. The principal suffering was, however, experienced at night, and especially after she had become warm in bed. The deep-seated "pain in the bone", would then entirely prevent her from sleeping. This condition had lasted, in a greater or less degree, for a year before she applied to King's College Hospital. Three years previous to this she had been treated for syphilis, and had taken mercury several times.

The joint was directed to be kept at rest, and three or four grains of the iodide of potassium were given three times a day. Under this treatment the swelling soon subsided, and the pain was much relieved. The joint could now be freely examined, and it became evident that the head of the tibia was the principal seat of the disease. Being relieved of her symptoms, the patient now discontinued her attendance, but remained free from pain for a short time only, when she again applied at the hospital: a repetition of the former treatment was again followed by complete relief. As soon as she discontinued her medicine, however, the symptoms returned. In this way she continued under treatment for three years, and at one time persevered with the iodide of potassium for six months without any material intermission. As long as she took the medicine she was easy, but upon discontinuing it the pain invariably recurred.

Being tired out by the long continuance of the disease, she now

1 The cases of trephining the tibia are in continuation of those previously given 
wished, if possible, that something more should be done, and I mentioned to her that permanent relief might possibly be afforded by trephining the head of the tibia, where, as I conceived, some local cause was keeping up the disease. The patient, who before had refused to go into the hospital, now readily consented, for the purpose of having the operation performed. On her admission, the left leg, round the head of the tibia, measured half an inch in circumference more than the right. There was comparatively little pain or swelling, as she had been taking her medicine for some days; but she complained of tenderness upon pressure about the insertion of the ligamentum patellæ, and over the upper part of the internal tuberosity of the tibia, which was evidently enlarged. As the shaft of the bone was in no way affected, and the pain and swelling were confined to the immediate neighbourhood of the joint, it appeared that the epiphysis of the bone had originally been the seat of the disease. It became, therefore, necessary to apply the trephine to this part, taking care, at the same time, to avoid injuring the articulation. I had the advantage of Mr. Fergusson's and Mr. Partridge's advice; the operation was performed with their concurrence.

On the 29th of Mas, a T-8haped incision having been made over the upper part of the internal tuberosity of the tibia, the parts below were carefully separated with the handle of the scalpel, until the bone was felt with the point of the finger. The trephine, which was made purposely, with a very deep blade, and not more than a third of an inch in diameter, was now applied. As soon as the outer shell of bone was perforated, the cancellous structure was felt to give way under the pressure of the instrument, and some minute and separate flakes of white matter were observed to escape with the blood by its side. After the operation, water dressing was applied, and an opiate ordered at night. On the first of June, the patient stated that her leg had not felt so easy for four years. She had slept well every night since the operation, and was free from pain. There was no fever.

On the 4th the knee felt stiff and sore, and rather uncomfortable, but she had had no return of the "old pain"; a gutta percha splint was placed behind the joint.

5th. She was again free from pain.

7th. There was now, for the first time, some return of what she distinguished as the old pain.

12th. All uneasiness had now subsided without any medicine, and she felt quite comfortable.

18th. Had again some discomfort about the knee, but no further return of the former pain. The puffy swelling on the outer side of the tibia, which had in a great measure subsided, now increased, and became painful upon pressure; a diseased gland in the neck at the same time began to enlarge. She was ordered some citrate of iron in combination with the small doses of the iodide of potassium, and was directed to get up, as it was supposed that her present symptoms depended in a great measure upon her confinement to bed. In a week after this time she was enabled to leave the hospital, when her general appearance rapidly improved.

On the 24th of August her health was quite restored; she could raise her leg without any pain or inconvenience, and had experienced no return of the " old pain" since leaving the hospital. 
The idea of trephining the tibia in this case was suggested by a case which occurred at the Lock Hospital, during the period when I held the office of house-surgeon. A young and delicate woman, after other symptoms which it is unnecessary to detail, became subject to intense and uninterrupted pain in the right thigh. The disease was relieved by none of the mcans employed, and the patient at length, after most protracted and severe suffering, died, apparently worn out by the pain. On making a section of the bone, I found that its cancellous structure was occupied at different parts by a morbid deposit. This occurred in irregular patches, completely filled the cancelli, and was of a light brown colour. It was moderately firm in consistence, and upon a chemical examination by Dr. Beale, of Carey Street, was pronounced to consist chiefly of fatty matter. The parietes of the bone were greatly thickened, and a kind of cancellous structure had been developed between the original outline of the bone and the newlyformed portions.

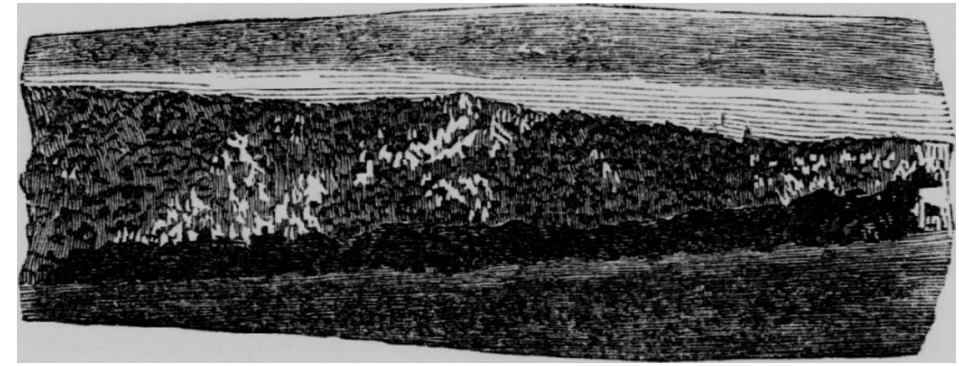

Fig. 6. Reprcsenting morbid deposit in bone.

The extreme distress which this patient endured arose probably from the tension produced by the interstitial deposit of bony matter: whether determined in this individual instance by the presence of diseased matter in the interior of the bone, or not, I will not undertake to say ; but, in either case, it appeared probable that the pain which constituted the really serious part of the disease might have been relieved, had a sufficient opening been made in the bone. If the morbid deposit kept up the irritation and produced the surrounding thickening, an artificial opening might at once have afforded relief. The object of such an opening would be not necessarily to remove all the diseased matter in the interior of the bone, but that it might be dissolved and expelled in the subsequent suppuration. If, on the other hand, the real disease were independent of the deposit in the cancellous structure, the removal of a portion of the dense and hard crust of the bone would be the means most likely to relieve the tension of the parts. Such were the considerations which determined me, should an opportunity present itself, to try the effect of making an artificial opening in cases of long-continued pain in bone, not yielding to internal remedies, and following the action of a morbid poison upon the system. In such an experiment there is everything to be gained and nothing to be lost. For should the operation entirely fail in removing the symptoms, the patient is not left in a worse condition than before.

The morbid deposit in the interior of bones to which I have alluded, may, I believe, take place so as to occupy a large portion of their 
cancellous structure; and that it then becomes a source of irritation in some cases, I cannot doubt, from the fact of openings being occasionally formed by a natural process of ulceration in bones long affected, as if to allow an exit for the morbid matter. These points are illustrated in the accompanying figure, for which $I$ am indebted to $\mathrm{Mr}$. Harrison, of the Albany Court-yard.

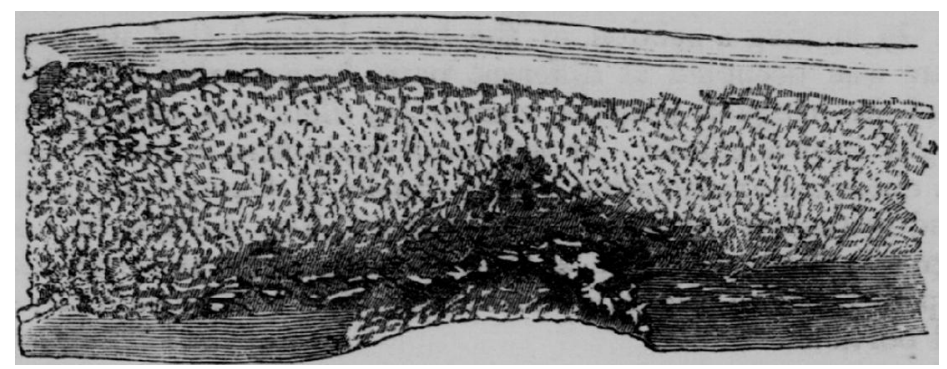

Fig. 7. Showing morbid deposit in bone, and ulcerated opening through its thickened wall.

In the class of cases now referred to, there may be no formation of pus in the bone. In this respect they differ from those previously mentioned. Independently, however, of the actual pressure of the confined fluid, the cases are strictly analogous. The essential characters of the disease may be the same, whether the original cause of local irritation arise from confined purulent fluid, or from a deposit of morbid matter in a more solid form, or from a piece of necrosed bone.

The following case, in which there was no suppuration and no external opening, presents an example of chronic irritation from necrosis, producing a very large deposit of new bone, and giving rise to some of the symptoms, although in a milder form, that were present in the cases of abscess in the tibia before related.

CAse IV. A. R., aged 24, was admitted into King's College Hospital, May 21, 1852. Twelve months previously, she had experienced much pain in the left leg. This was attended with swelling and redness extending over the whole limb. After a time, she went into Guy's Hospital, where she remained twenty-two weeks, and then returning home, continued without attendance for three months.

On admission into King's College Hospital, the two lower thirds of the left tibia were very much enlarged; the surrounding skin was red and tender upon pressure; she complained of pain in the leg, which at night was occasionally sufficiently severe to cause her to get up and walk about the room. Generally speaking, she found that the leg was easier when hanging down than at other times. There was no external opening, nor any apparent constitutional or hereditary disease to account for the symptoms. She was kept in bed for five weeks, and some internal medicines were given; but as no benefit appeared to be derived from these means, the operation of trephining the tibia was performed upon the 1st of July. It became apparent during the operation that the bone was very greatly thickened and condensed; and it was with some difficulty that the trephine was made to perforate its dense substance. When the first portion of bone was removed, the irregular rough surface of some dead bone could be felt beneath. It became therefore evident that the case was one of necrosis. The tre- 
phine, which was of small diameter, was now applied to different parts, 80 as to admit of a considerable portion of the newly-formed bone being removed by sawing between the openings. These would represent the corners, and the lines of the saw the sides, of an oblong. The portion of bone thus raised was half an inch thick at its thinnest part. Between it and the exposed and necrosed shell of the tibia there was no fluid whatever. A portion of the dead bone at the bottom of the wound was now removed in a similar way, and in the centre of the old bone was found a condensed and detached portion of dead cancellous structure. It is unnecessary to give any further details of this case, except to mention that, after the operation, the pain, as well as the redness of the skin of the leg, entirely subsided, and in less than two months she was sufficiently well to leave the hospital.

Strumous deposit in bone may in like manner become the source of chronic irritation and long continued pain. It is seldom, indeed, that crude tubercle is deposited in any quantity in bone, but the following case, for which I am indebted to Mr. Hewett, of St. George's Hospital, will serve to shew that when it does occur the symptoms will bear a certain resemblance to those of chronic abscess. A man was admitted into St. George's Hospital with a tumour situated at the union of the middle to the lower third of the thigh. This tumour was unyielding in its nature and not painful when handled. Fifteen months previously the patient had begun to suffer considerable pain in the bone, of a shooting character. This deprived him of rest at night. Three months afterwards the swelling made its appearance, and gradually increased in size; seven months from the time he was first attacked his health began to fail, and the pain in the thigh had, with few intermissions, been continual and of a severe character. The year following his first admission into the hospital, he was attacked with erysipelas, and died. On examining the thigh, great thickening and condensation. were observed between the muscles and the bone. The periosteum, which was much thickened, presented, on its free surface, a large patch of tubercular matter, enveloped in a dense cyst. The bone itself was irregular in shape, much hypertrophied, and very hard. Its medullary cavity at this point was filled with tubercular matter, surrounded by gray semitransparent lymph. Deposits of scrofulous matter were also found in other parts.

From the examples now adduced it appears that long continued pain in bone may arise from a variety of different pathological conditions, and that the chronic irritation, which precedes the deposit of new bone, may depend, among others, upon the following local causes :-1. Upon the formation of pus within the bone. 2. Upon the deposition there of more solid material arising from the poisons of mercury, or syphilis. 3. Upon a collection of tubercular matter in bone; or 4, upon the presence of a necrosed portion of cancellous structure.

When the source of the continued irritation is thus situated within the bone itself, it seems not unreasonable to conclude that a similar plan of treatment would be available, from whatever cause that irritation may arise; and the results hitherto obtained would appear to lead to the inference that relief may be expected from an artificial opening in several classes of cases.

Wherever there is reason to suspect that pain in a bone is kept up 
by the presence of some morbid or foreign matter in its interior, or by the pressure produced by a redundancy of bony deposit, it appears evident that the removal of a piece of the shell of the bone is the rational mode of treatment. An opportunity is thereby afforded at once for the escape of any confined matter, and the tension of the parts is relieved; and it appears not improbable, from the favourable effects hitherto obtained by this mode of treatment, that it may hereafter be extended to the relief of many cases of protracted and obscure affection of the osseous system.

13, Dover Street, Piccadilly, September 1852.

\section{BIBIIOGRAPEICAI RECORD.}

The Physicat Diagnogis of Digfages of the Abdomen. By Edward BALLARD, M.D.Lond. pp. 276. London : 1852.

Dr. BaLnarD's work is divided into three parts, the first of which treats of the Method and Genkral Results of Physical Examination; the

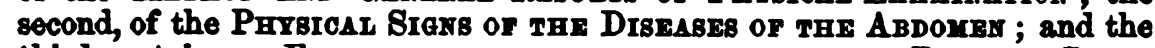
third contains an Exumeration or the most bemarkable Physical Sighs,

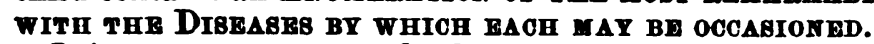

It is necessary to remark, that Dr. Ballard has included in his work the Physical Diagnosis of the Diseases of the Pelvis, for reasons which appear to us to be quite satisfactory, for, as he justly observes, the viscera of the pelvis often rise up so as to occupy the cavity of the abdomen; and, on the other hand, not only are some of the healthy abdominal structures prolonged into the pelvis, but the products of diseased action gravitate into this carity. The introduction of the Physical Diagnosis of Pregnancy, although not a disease, is also, in our opinion, quite justifiable, and the directions given for the digital examination of the pelvic organs by the vagina and rectum add very much to the interest and importance of Dr. Ballard's treatise.

Five methods of physical examination are described by Dr. Ballard, vir., ImsPBorion, by which much valuable information is obtained as to the positive and relative size, and as to the situation and movements of the abdominal contents; Massoratros, by which an accurate result is afforded in confirmation of the knowledge gained by inspection; PAIPAmIOn, by which the size, situation, consistence, and other properties of organs may be estimated; Precussion, which offers results in the abdominal organs of no less value than it jields in those of the thorax; and ADscolrarion, which although of comparatively limited application in the investigation of abdominal disease, jet in certain special circumstances, as in the determination of pregnancy and of arterial tumours, affords information of unquestionable importance.

The following extract on the mode of inspecting the abdomen is of great practical value.

"In accordance with my former remarks, inspection must take in simultaneously the abdomen and the chest. Both must of course be uncorered and exposed in good light, the subject being protected from cold by a previous regulation of the temperature of the room. This is essential, not only for the benefit of the person examined, but because a sensation of chilliness is incompatible with that perfect rest which it is desirable that the abdominal and thoracic muscles should maintain during the period of inspection. The position of the subject may be either the erect or the recumbent. 